\title{
Existence of bound states of a polaron with a breather in soft potentials
}

\author{
J. Cuevas, ${ }^{1}$ P. G. Kevrekidis, ${ }^{2}$ D. J. Frantzeskakis, ${ }^{3}$ and A. R. Bishop ${ }^{4}$ \\ ${ }^{1}$ Grupo de Física No Lineal, Departamento de Física Aplicada I, Escuela Universitaria Politécnica, C/ Virgen de África, 7 , \\ 41011 Sevilla, Spain \\ ${ }^{2}$ Department of Mathematics and Statistics, University of Massachusetts, Amherst, Massachusetts 01003-4515, USA \\ ${ }^{3}$ Department of Physics, University of Athens, Panepistimiopolis, Zografos, Athens 15784, Greece \\ ${ }^{4}$ Theoretical Division and Center for Nonlinear Studies, Los Alamos National Laboratory, Los Alamos, New Mexico 87545, USA
}

(Received 23 December 2005; revised manuscript received 4 June 2006; published 16 August 2006)

\begin{abstract}
We consider polarons in models of coupled electronic and vibrational degrees of freedom, in the presence of a soft nonlinear substrate potential (Morse potential). In particular, we focus on a bound state of a polaron with a breather, a so-called "polarobreather." We analyze the existence of these states based on frequency resonance conditions and illustrate their stability using Floquet spectrum techniques. Multisite solutions of this type are also obtained both in the stationary case (bond-centered and twisted polarons) and in the breathing case (bond-centered and twisted polarobreathers). For all the branches examined, the dynamical evolution of instabilities pertinent to the corresponding solutions are also briefly discussed. Finally, a different branch of socalled phantom polarobreathers is also demonstrated.
\end{abstract}

DOI: 10.1103/PhysRevB.74.064304 PACS number(s): 63.20.Pw, 63.20.Ry, 63.20.Kr, 05.45.-a

\section{INTRODUCTION}

In the past few years, the study of discrete nonlinear systems has received a great deal of attention, chiefly due to the increasing number of pertinent physical applications. ${ }^{1}$ Among the diverse areas of recent interest are coupled waveguide arrays in nonlinear optics, ${ }^{2}$ Bose-Einstein condensates (BECs) trapped in deep optical lattices $(\mathrm{OLs})^{3}$ in atomic physics, coupled cantilever systems in nanomechanics, ${ }^{4}$ the local denaturation of the DNA double strand in biophysics, ${ }^{5}$ stellar dynamics in astrophysics, ${ }^{6}$ and so on.

In the study of such discrete nonlinear systems, an important concept is the possibility of intrinsic localization due to interacting degrees of freedom, even in a linear regime, as proposed by Holstein. ${ }^{7,8}$ This type of system has been shown to sustain single- and multihumped polaronic and excitonic solutions. ${ }^{9}$ The same notion of effective nonlinearity in the setting of coupled excitonic and vibrational degrees of freedom was central to Davydov's suggestion of solitonic excitations arising in biomolecules. ${ }^{10,11}$ More recently, a new aspect has been added to this type of problem ${ }^{12,13}$ by considering the interplay of the linear self-trapping with a soft nonlinear potential, such as a Morse potential. This has been proposed as a more general model relevant to many soft matter applications.

In the context of these earlier works, it was found that single-site, as well as multisite, polarons can exist and may be stable. Such solutions, however, terminate in saddle-node bifurcations, when continued as a function of appropriate parameters of the system (such as the coupling strength of the electronic-vibrational degrees of freedom). On the other hand, in a different context, breathing excitations of electronic-vibrational models were discussed in Ref. 14 and were thereby termed polarobreathers. This was proposed in the setting of a cubic anharmonic potential and polarobreathers were found to be potentially stable. However, instability due to resonance mechanisms of the mode's spectrum with multiples of its frequency were also briefly discussed.
Our purpose in the present paper is to examine the soft nonlinear model with the Morse potential and to provide a number of insights regarding its solutions and their stability. We start by establishing that the main stationary solutions obtained earlier in Ref. 12 (namely, the single-site polarons) are dynamically stable within their region of existence. We then turn our attention to the genuinely "breathing" (in time) solutions of the model (the so-called polarobreathers). We will obtain (and justify, based on resonance conditions) the domain of existence of such solutions. We will also systematically investigate their stability by performing the corresponding Floquet spectral analysis and examine the linear stability of these solutions. Our study of this new class of solutions will reveal an additional family of solutions consisting of a polarobreather combined with a linear mode, in the form of a "phantom polarobreather." Since the branch of stationary polarons terminates at a critical value of the coupling, as illustrated in Ref. 12, we will examine the dynamics beyond this termination point with a single hump initial condition and show that the solution develops large breathing fluctuations in its local (i.e., central site) energy due to an interplay between the central site of the localized profile and its neighbors. We will also highlight multisite branches such as the two-site stationary solutions (polarons) and the twosite polarobreathers and numerically study their stability.

Our presentation is structured as follows. In Sec. II, we present the model and numerical methods (the details of which are relegated to a technical appendix). Subsequently, in Sec. III, we present and discuss our numerical results. Finally, in Sec. IV, we summarize our conclusions and pose questions of interest for future studies.

\section{THE MODEL}

We consider the coupled charge/excitation-lattice model introduced in Ref. 12, describing the competition between linear polaronic self-trapping and self-focusing effects of a 
soft nonlinear potential; in dimensionless form, it can be expressed as follows:

$$
\begin{gathered}
i \dot{\Psi}_{n}=-J\left(\Psi_{n+1}+\Psi_{n-1}\right)-\chi u_{n} \Psi_{n}, \\
\ddot{u}_{n}=-V^{\prime}\left(u_{n}\right)+\chi\left|\Psi_{n}\right|^{2}+k\left(u_{n+1}-2 u_{n}+u_{n-1}\right),
\end{gathered}
$$

where dots denote time derivatives, and the lattice index $n$ runs from 1 to $N$ (the total number of lattice sites). In Eqs. (1) and (2), $\Psi_{n}(t)$ represents the "electronic" degrees of freedom, $u_{n}$ corresponds to the lattice displacements (i.e., "vibrational" degrees of freedom), while the parameters $J, k$, and $\chi$ denote, respectively, the transfer integral, the lattice spring constant, and the coupling constant between the interacting fields. Finally, $V\left(u_{n}\right)$ is an anharmonic on-site potential, which, as in Ref. 12, is assumed to have the form of a Morse potential:

$$
V(u)=\frac{1}{2}[\exp (-u)-1]^{2} .
$$

The dynamical equations (1) and (2) arise from the Hamiltonian:

$$
\begin{aligned}
H= & \sum_{n}\left[\frac{1}{2} \dot{u}_{n}^{2}+V\left(u_{n}\right)+\frac{k}{2}\left(u_{n}-u_{n+1}\right)^{2}\right] \\
& -\sum_{n}\left[\chi\left(\left|\Psi_{n}\right|^{2} u_{n}\right)+J\left(\Psi_{n} \Psi_{n+1}^{*}+\text { c.c. }\right)\right] .
\end{aligned}
$$

We will seek standing-wave as well as genuinely timeperiodic solutions of Eqs. (1) and (2). To factor out the phase invariance of Eq. (1), and clearly distinguish between the two classes of solutions, we introduce the transformation:

$$
\Xi_{n}(t)=\Psi_{n}(t) e^{-i \omega_{e} t} .
$$

Then, the dynamical equations take the following form:

$$
\begin{gathered}
i \dot{\Xi}_{n}-\omega_{e} \Xi_{n}+J\left(\Xi_{n+1}+\Xi_{n-1}\right)+\chi u_{n} \Xi_{n}=0, \\
\ddot{u}_{n}+V^{\prime}\left(u_{n}\right)-\chi\left|\Xi_{n}\right|^{2}-k\left(u_{n+1}-2 u_{n}+u_{n-1}\right)=0 .
\end{gathered}
$$

It is worthwhile noting that the $\mathrm{U}(1)$ invariance of the first of the above dynamical equations immediately implies a phase freedom, which, in turn, leads to the conservation law of the mass $\left(l^{2}\right.$ norm) of the electronic field.

Stationary solutions of the above system correspond to ones where all time derivatives in Eqs. (6) and (7) are set to zero. On the other hand, time-periodic solutions are characterized by a frequency of oscillation $\omega_{\mathrm{b}}$ (or period $T_{\mathrm{b}}$ $\left.=2 \pi / \omega_{\mathrm{b}}\right)$ in the time dependence of both the electronic wave function $\Xi_{n}$ and of the lattice displacements $u_{n}$. Our aim is to study the existence and stability of such stationary and breathing polaron solutions; the latter, adopting the terminology of Ref. 14, will be henceforth called "polarobreathers." In the results to be presented below, we have fixed the values of the transfer integral and lattice spring constant, namely $J=0.005$ and $k=0.13$, and let the coupling constant $\chi$ and the polarobreather frequency $\omega_{\mathrm{b}}$ vary. We have also considered other parameter values (such as, e.g., $k=0.065, k=0.26, J$ $=0.0025$, and $J=0.01$ ) and have obtained qualitatively similar results to those reported below.
In order to find either stationary or time-periodic solutions of the dynamical equations (6) and (7), we use methods based on the anticontinuum limit. ${ }^{15,16}$ In particular, we first obtain a nontrivial steady state $u_{0}$-for stationary solutions-or an orbit of frequency $\omega_{\mathrm{b}}$ for an isolated oscillator $u_{0}(t)$-for time-periodic solutions. Then, the solution at the anticontinuum limit $(k=J=\chi=0)$ is $\Xi_{n}=u_{n}=0$, except at $n=0$, which is set to $u_{0}$. The coupling constants are subsequently varied through a path-following (Newton-Raphson) method. In the following, we first vary $\chi$ up to 0.02 , then $J$ up to 0.005 and, finally, $k$ up to 0.13 . Once $J$ and $k$ are fixed, $\chi$ can be freely varied. The implementation of the anticontinuum limit has been performed both in real space (using a shooting method) and in Fourier space. Note that the first method is less accurate than the second one, whereas the latter is rather time-consuming, particularly in the case of long chains. We have made use of both methods in the calculations presented herein.

Once the numerically exact [up to the prescribed accuracy $\mathrm{O}\left(10^{-7}\right)$ ] solutions are obtained, linear stability analysis is performed to examine the dynamical stability of the solutions. In the case of stationary solutions, this is implemented by imposing normal mode perturbations (see, e.g., earlier work in Refs. 8, 13, and 17) and obtaining the corresponding eigenvalues of the ensuing linear matrix problem. On the other hand, in the case of (time-periodic) polarobreather solutions, we impose time-dependent perturbations to both the electronic and vibrational fields and solve the resulting (linear) differential equations for the perturbations from $t=0$ to $t=T_{b}$. Then, obtaining the Floquet (or monodromy) matrix, which relates the perturbation vector at $t=0$ to that at $t=T_{b}$, we compute the Floquet multipliers of the periodic solution. The Floquet multipliers imply stability (instability), if they do (do not) appear only on the unit circle. The details of the numerical methods used in this work are discussed in the Appendix.

\section{NUMERICAL RESULTS}

In this section we illustrate the numerical results obtained for the polarobreathers. Recall that in all calculations we have fixed $J=0.005$ and $k=0.13, \chi$ being a free parameter.

We first consider the stability of static polarons. Such polarons, localized on a single lattice site, turn out to be always stable. Considering perturbations of these structures, namely $u_{n}(0)=u_{n}^{0}(0)+\varepsilon \delta_{n, 0} \quad$ and $\quad \operatorname{Re}\left[\Psi_{n}(0)\right]=\operatorname{Re}\left[\Psi_{n}^{0}(0)\right]+\varepsilon \delta_{n, 0}$ (where the zero superscript denotes an exact solution), it is found that the polaron evolves into a vibrating state, which is a normal mode of the system. This result is clearly illustrated in Fig. 1 (here we have used $\varepsilon=0.001$ and $\chi=0.5$ ); note that in the relevant Fourier spectrum [middle and bottom panels of the same figure for the vibrational (middle) and electronic (bottom) field at the central site], there appears a single excited frequency.

Apart from static polarons, there exist stable, timeperiodic and spatially localized solutions within the context of the model, namely polarobreathers. One-site and two-site (see below for details on the latter) such solutions can be continued as a function of $\chi$ for $J$ and $k$ fixed; other para- 

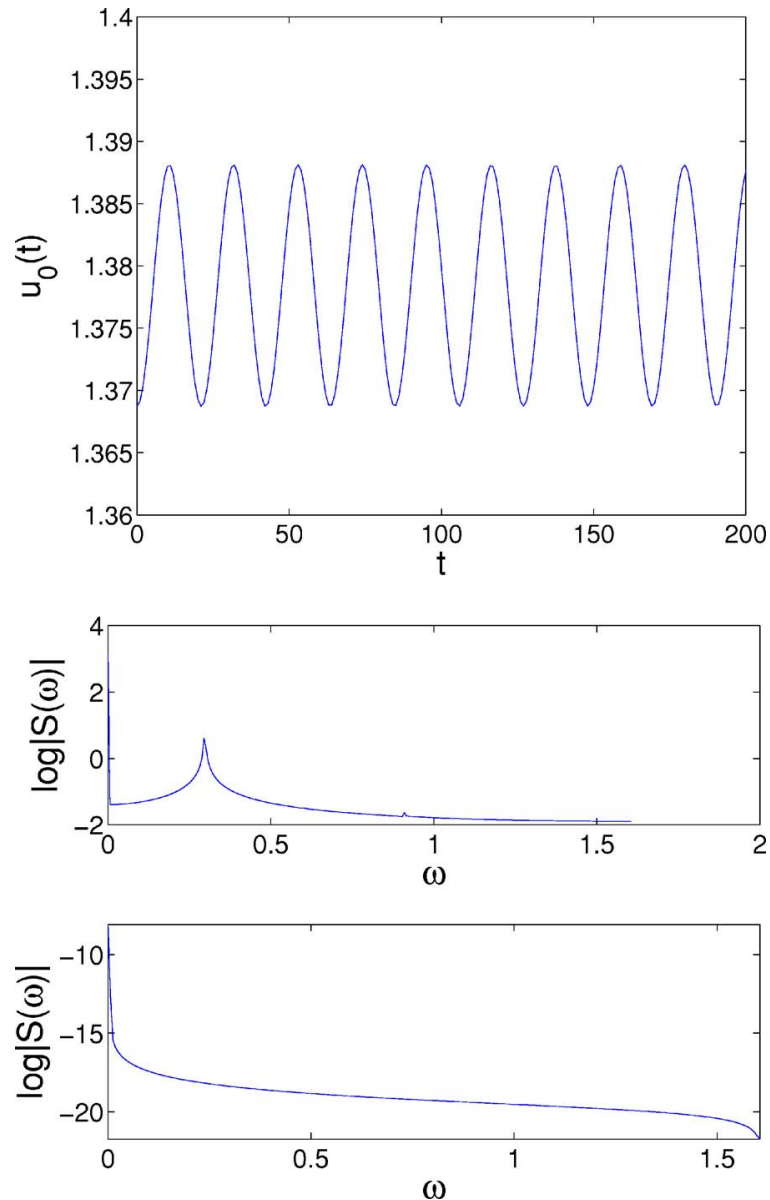

FIG. 1. (Color online) Top panel: Evolution of the displacement of the central particle of a slightly perturbed static polaron with $\chi$ $=0.5$. Middle and bottom panels: The Fourier spectrum of the corresponding time series for the lattice variable (middle) and the electronic one (bottom). The existence of only one frequency indicates the simple periodic nature of the solution.

metric variations can, of course, also be performed. The domains of existence of the one-site and two-site polarobreathers for $k=0.13$ and $J=0.005$ are shown in Fig. 2. An example

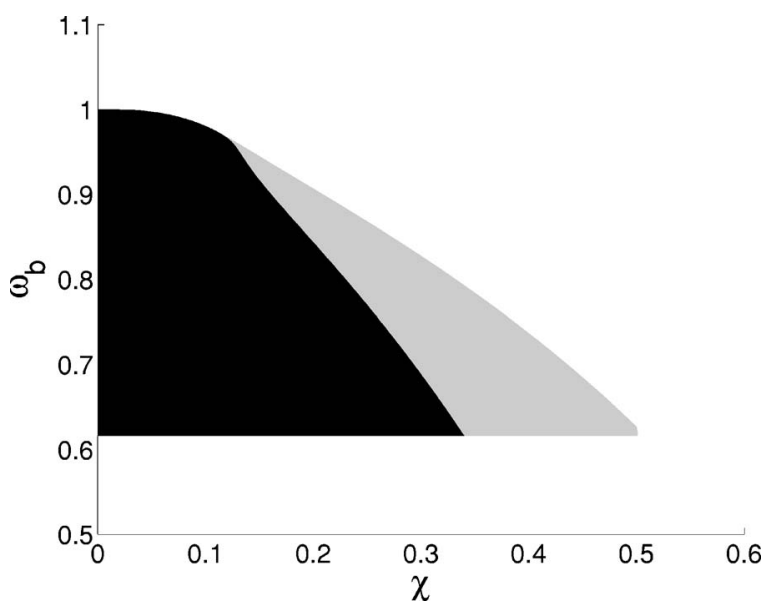

FIG. 2. Domain of existence of one-site (black region) and twosite (black and gray region) polarobreathers in the $\chi-\omega_{\mathrm{b}}$ parameter plane.
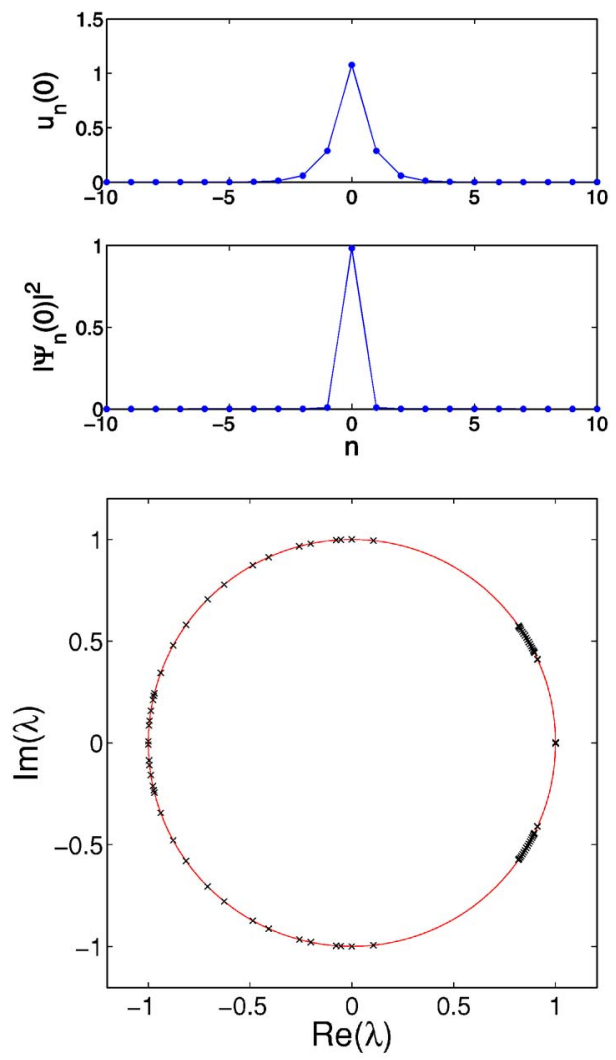

FIG. 3. (Color online) Top and middle panels: Profiles of the lattice $\left(u_{n}\right.$, top) and electronic component $\left(\left|\Psi_{n}\right|^{2}\right.$, middle $)$ of a one-site polarobreather with $\omega_{\mathrm{b}}=0.8$ and $\chi=0.15$. Bottom panel: Floquet spectrum of this solution.

of a one-site polarobreather solution, along with the relevant Floquet spectrum, is illustrated in Fig. 3. Additionally, Fig. 4 shows the Fourier spectrum of this solution, whose nonlinear character implies the existence of integer multiples of its frequency. The time evolution of the lattice $\left[u_{0}(t)\right]$ and electronic $\left[\Psi_{0}(t)\right]$ variables of the central particle is displayed in Fig. 5. Notice that the oscillation periods of the fields $u$ and
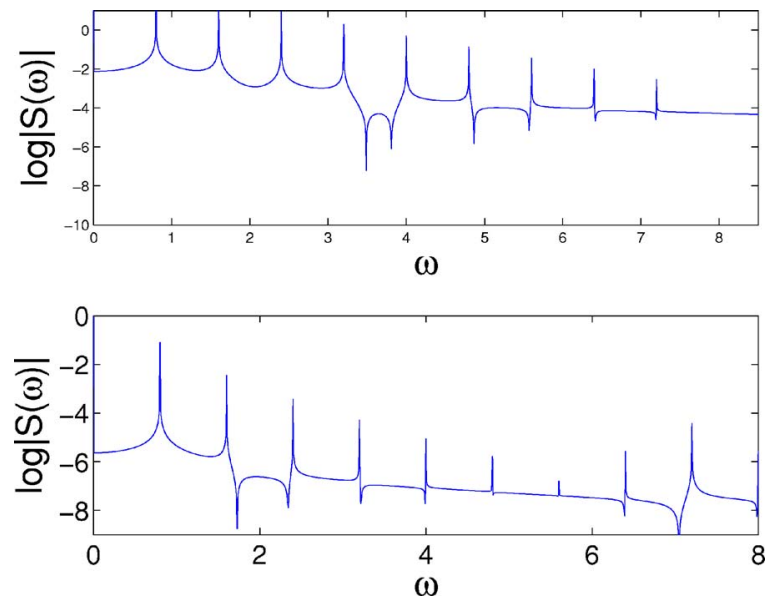

FIG. 4. (Color online) Fourier spectrum of the polarobreather of Fig. 3. The top and bottom panels correspond to the lattice and electronic variables, respectively. 

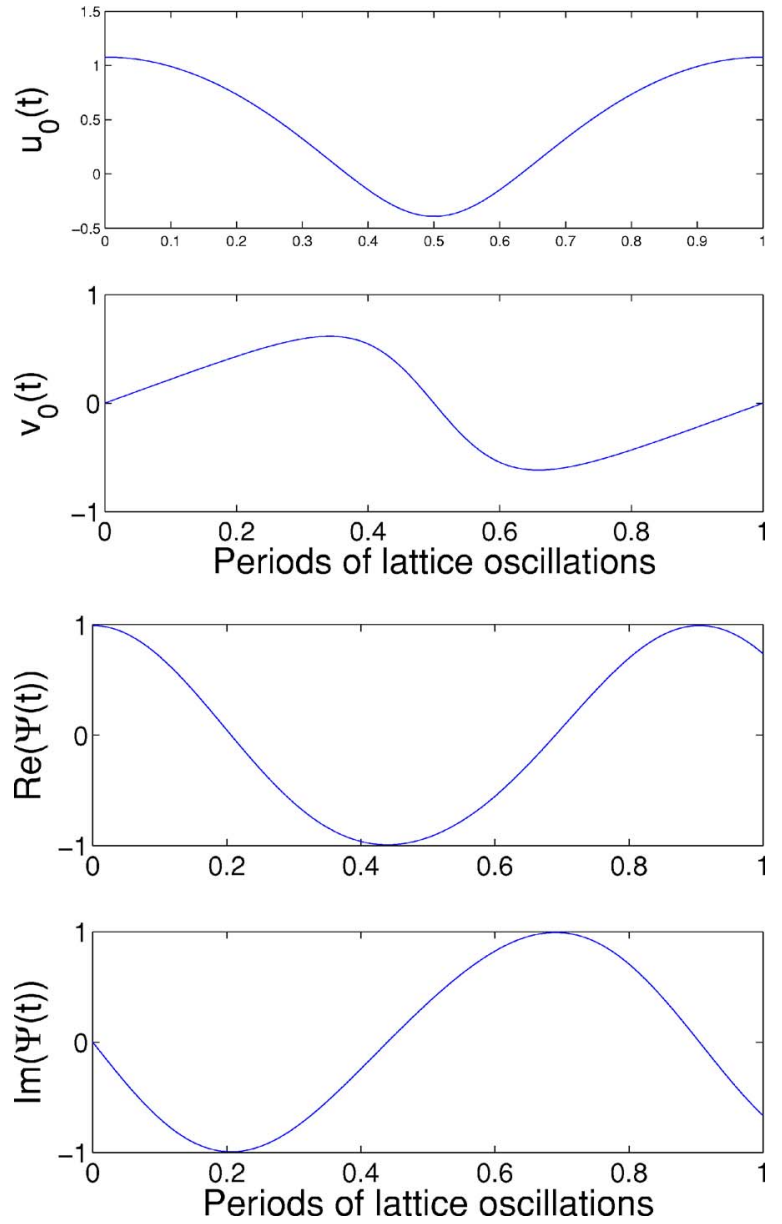

FIG. 5. (Color online) Time evolution of the lattice (top panels) and electronic coordinates (bottom panels) of a polarobreather with $\omega_{\mathrm{b}}=0.8$ and $\chi=0.15$. Notice that the oscillation period of the two functions is different (see text).

$\Psi$ are different, due to the additional inclusion (in $\Psi$ ) of the oscillation with frequency $\omega_{e}$. Polarobreathers exist as long as the conditions of the MacKay-Aubry's theorem ${ }^{15}$ are fulfilled, i.e., none of the harmonics of the polarobreather frequency resonate with the linear modes. Based on this criterion, an analysis of the linear modes can provide the range of existence of polarobreathers of a given frequency. Figure 6 shows the real part of the normal mode frequencies and indicates the existence of a continuum band of (extended) linear modes in addition to several localized modes. Since we are dealing with polarobreathers in a soft potential, they must stem from the linear mode at the bottom of the band of extended modes. This mode becomes localized (i.e., bifurcates from the continuous spectrum band) at the value of $\chi$ $\approx 0.033$ and its location varies with $\chi$. As a result, the polarobreather frequency must be smaller than this localizedmode frequency. This fact is indicated in the bottom panel of Fig. 6; it can be observed that the bifurcation points correspond to the values of $\chi$ for which the frequency of the localized mode coincides with the frequency of the polarobreather branch.

From Fig. 6 it can be inferred that the higher the frequency of the polarobreather is, the closer this solution is to
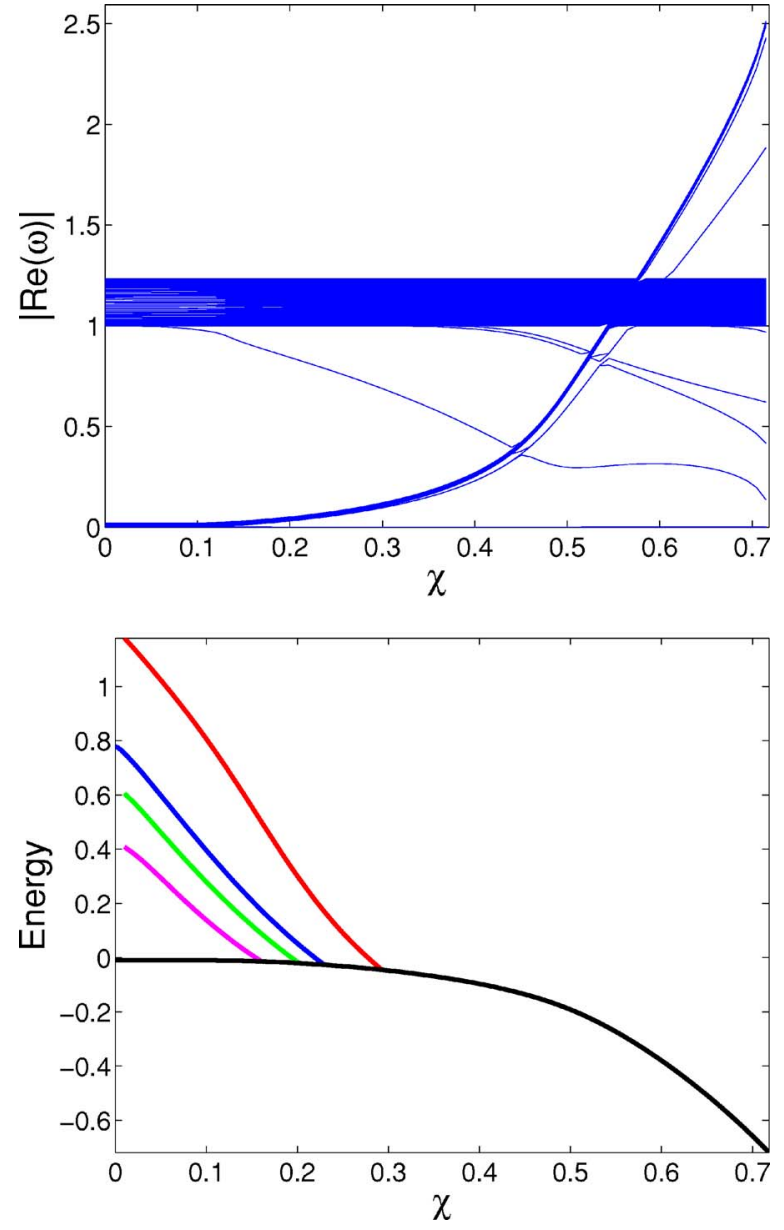

FIG. 6. (Color online) The top panel shows the real part of the linear mode spectrum. The bottom panel shows the polarobreather energy as a function of $\chi$ for different values of the frequency; from left to right: $\omega_{\mathrm{b}}=0.9,0.84,0.8$, and 0.7 . The line at the bottom corresponds to the static polaron.

a static polaron. This result seems counterintuitive, as the static polaron has zero frequency and the polarobreather closest to the polaron should be the smallest frequency one. This "paradox" can be resolved as follows: The polaron is actually a deformation in the lattice due to the existence of an electron. A polarobreather is an oscillation around these nontrivial vibration equilibrium positions, which has nonzero action I-phase-space area (see Refs. 18 and 19). Consequently, a static polaron is a zero action polarobreather, rather than a zero frequency one. Furthermore, the polarobreather that becomes a polaron in the limit $\chi \rightarrow 0$ is the one whose frequency approaches to the smallest within the continuum band of the linear modes spectrum (that is, $\omega_{\mathrm{b}} \rightarrow 1$ ); this is due to the fact that the action of the polarobreather tends to zero when it approaches to the continuum band. This phenomenon has been previously observed in Refs. 20 and 21 and is illustrated in Fig. 7; it is clear that, for a given value of $\chi$, the higher the frequency is, the smaller the action is.

The existence of polarobreathers is also limited by the second-harmonic resonances. In the case of finite lattices, "phantom" solutions can appear due to the existence of gaps 

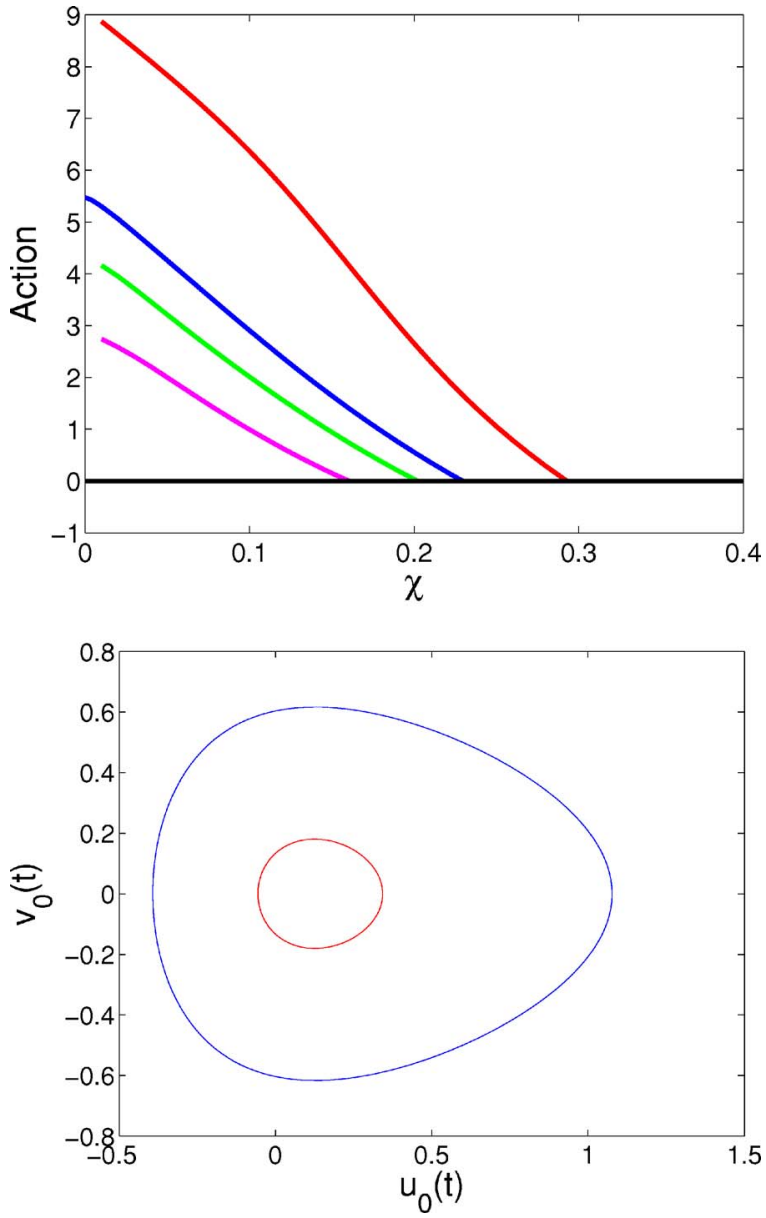

FIG. 7. (Color online) The top panel shows the polarobreather action as a function of $\chi$ for different values of the frequency; from left to right: $\omega_{\mathrm{b}}=0.9,0.84,0.8$, and 0.7 . The line at $I=0$ corresponds to the static polaron. The bottom panel shows the phase space coordinates for the central particle of a polarobreather with $\omega_{\mathrm{b}}=0.8$ (outer curve) and $\omega_{\mathrm{b}}=0.9$ (inner curve); in both cases $\chi=0.15$.

in the extended modes band. ${ }^{22}$ These solutions comprise a core that vibrates with the fundamental frequency $\omega_{\mathrm{b}}$ (or may also be static), whereas the tails correspond to a phonon vibrating with frequency $2 \omega_{\mathrm{b}}$. These solutions are an artifact of the finiteness of the lattice and, moreover, they are usually unstable. An example of a phantom polarobreather is shown in Fig. 8 (for $\omega_{\mathrm{b}}=0.6$ and $\chi=0.15$ ).

An analysis of the stability of polarobreathers is shown in Fig. 9. It is observed that, for the considered branch, all the solutions are linearly stable. It is worth noticing that for every polarobreather, two pairs of phase/growth modes exist.

Apart from one-site (site-centered) polarons, we have also considered intersite (bond-centered) static polarons and polarobreathers. Contrary to the case of the one-site polarons which are stable, intersite ones are unstable. An example is shown in Fig. 10, where the real and imaginary parts of the linear mode spectrum for bond-centered polarons are illustrated.

Similarly to their static counterparts, intersite polarobreathers are unstable. This instability is exponential (i.e., the Floquet eigenvalues responsible for the instability have zero
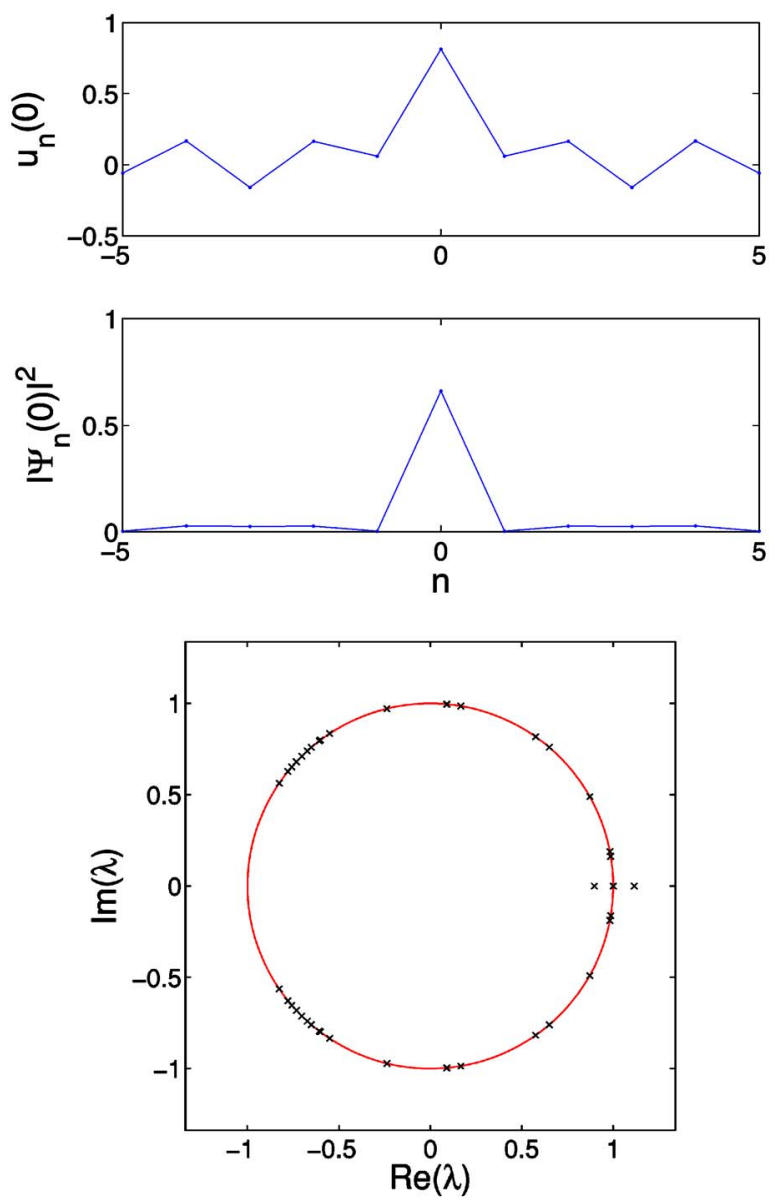

FIG. 8. (Color online) Top and middle panels: The profiles of the lattice (top) and the electronic (middle) components of a phantom polarobreather are shown for $\omega_{\mathrm{b}}=0.6$ and $\chi=0.15$. Bottom panel: Floquet spectrum of this (unstable) solution.

phase). In Fig. 11, an example of a two-site polarobreather, together with its Floquet spectrum, is shown. A full stability analysis for a branch of solutions is shown in Fig. 12. Notice that this instability (likewise for intersite polarons and polarobreathers) is due to the minimax nature of these solutions, a feature which arises even at the level of the regular Holstein model.

It is interesting to note that, similarly to what occurs for their static counterparts, ${ }^{12}$ one-site polarobreathers have a narrower domain of existence (black region in Fig. 2) than their intersite counterparts (gray and black region in Fig. 2). Given that the termination of such branches occurs upon their collision with the stationary branch of solutions, this trait is rather natural in the present setting. A wider domain of existence for two-site breathers was also observed in a model with competing attractive and repulsive interactions. ${ }^{23}$

In order to examine the dynamical evolution of the instability of intersite polarons, we again consider the perturbation $u_{n}(0)=u_{n}^{0}(0)+\varepsilon \delta_{n, 0}$ and $\operatorname{Re}\left[\Psi_{n}(0)\right]=\operatorname{Re}\left[\Psi_{n}^{0}(0)\right]+\varepsilon \delta_{n, 0}$, with the value $\varepsilon=0.001$. As illustrated in Fig. 13, after a transient, the polaron evolves into an antiphase vibrating state; note that if the two central particles were perturbed, the observed behavior would be similar, but the transient would be longer. If the same perturbation is applied to a two-site 

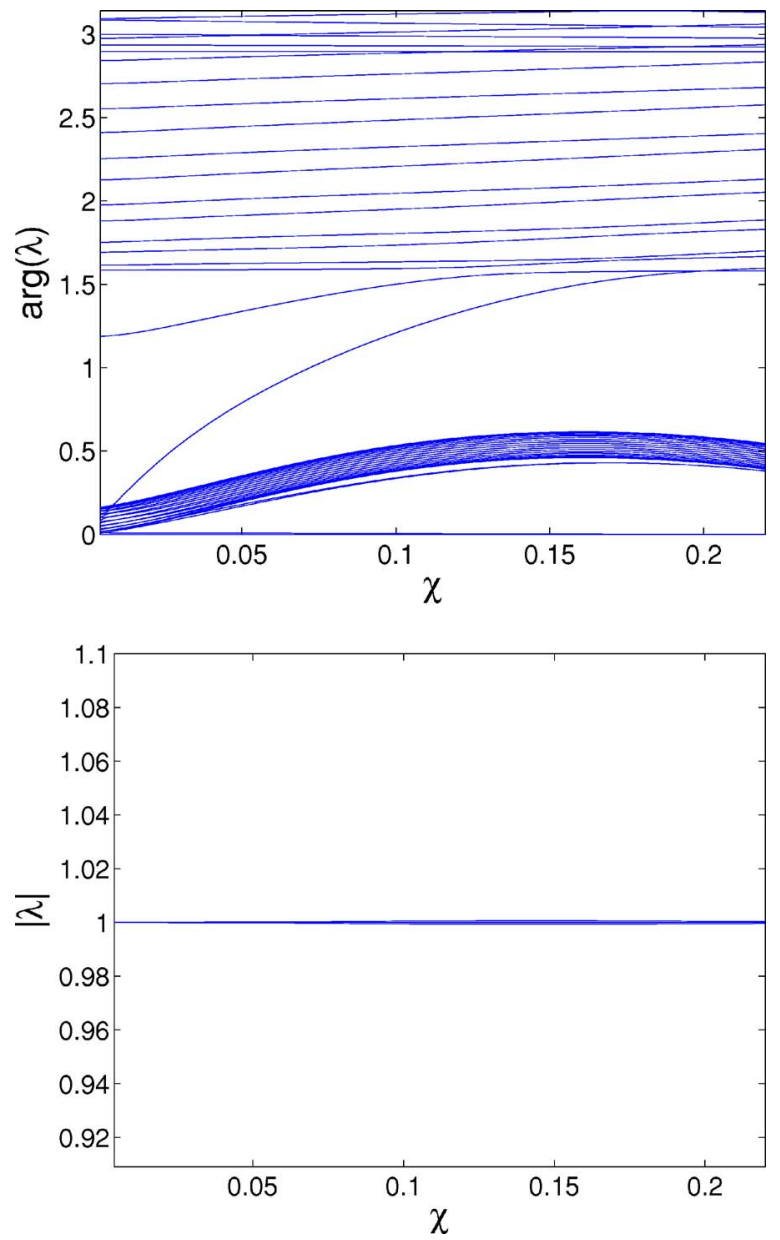

FIG. 9. (Color online) The argument (top) and modulus (bottom) of the Floquet eigenvalues as a function of the coupling constant $\chi$ for one-site polarobreathers.

polarobreather, the behavior is somewhat reminiscent of that observed for a static polaron. This is illustrated in Fig. 13 upon displaying the temporal evolution of the energy density at the two principal sites of the solution. The energy density is defined as

$$
\begin{aligned}
e_{n}= & \frac{1}{2} \dot{u}_{n}^{2}+V\left(u_{n}\right)+\frac{k}{4}\left[\left(u_{n}-u_{n+1}\right)^{2}+\left(u_{n}-u_{n-1}\right)^{2}\right]-\chi\left|\Psi_{n}\right|^{2} u_{n} \\
& -\frac{J}{2}\left(\Psi_{n}\left(\Psi_{n+1}^{*}+\Psi_{n-1}^{*}\right)+\text { c.c. }\right),
\end{aligned}
$$

where the asterisk (and c.c.) denotes a complex conjugate.

We have also analyzed the existence and stability properties of twisted polarobreathers, i.e., polarobreathers with an antiphase pattern in the electronic function, and an in-phase (type I) or an antiphase (type II) pattern in the vibrational variables. The profiles of the lattice and the electronic components of a type I and a type II twisted polarobreather are shown in Fig. 14. These solutions are a generalization of the corresponding twisted localized modes (see, e.g., the recent analysis of Ref. 24 and references therein). As shown in Fig. 15 , both types of solutions bifurcate from a static polaron branch, with the polaron having antiphase electronic vari-
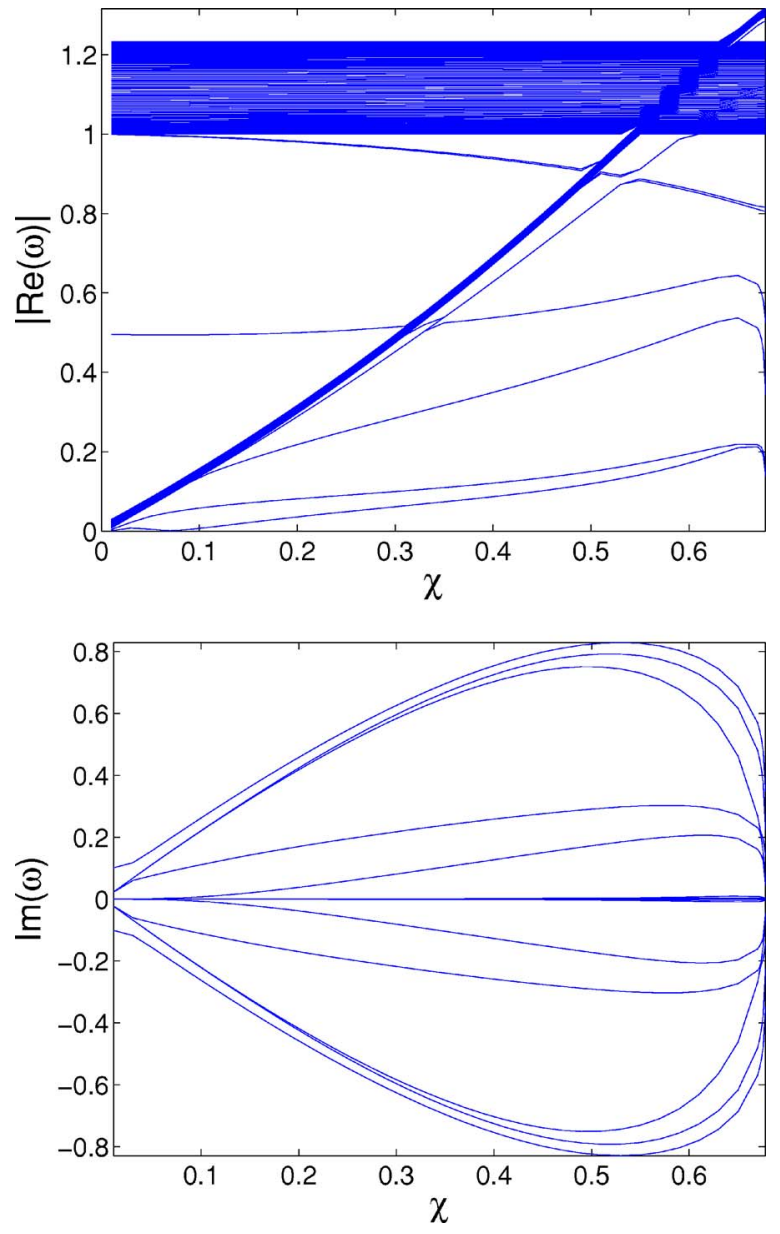

FIG. 10. (Color online) The real (top) and imaginary (bottom) parts of the linear mode spectrum as a function of the coupling constant $\chi$ for intersite polarons.

ables and in-phase lattice variables. The origin of this bifurcation lies in the coincidence of the frequency of the polarobreather with the frequencies of the linear localized modes detaching from the extended ones (see Fig. 16).

We can summarize the results for the stability of the twisted polarobreathers (and polarons) as follows.

Type I twisted polarobreathers are unstable due to oscillatory instabilities (i.e., the Floquet eigenvalues responsible for the instability have phases different from zero or $\pi$ ) as long as $\chi<0.277$. Beyond this critical value, solutions are linearly stable, as shown in Fig. 17.

Type II twisted polarobreathers suffer the same type of instability for $\chi<0.107$ and $\chi \in[0.125,0.367]$. They are also exponentially unstable for $\chi \in[0.151,0.414]$. Outside these intervals, the polarobreathers are stable, see Fig. 18.

Twisted polarons only exist for $\chi \geq 0.224$. They are stable except for the interval $\chi \in[0.224,0.303]$.

Another interesting fact is that the static polaron branch does not exist for $\chi>\chi_{c} \approx 0.718$. In particular, if the solution for a polaron close to $\chi_{c}$ is used as the initial condition for $\chi>\chi_{c}$, e.g., for $\chi=0.72$, we have found the following type of evolution (see Fig. 19): Initially and for some finite time, a continuous increase of the "local" energy at the central site of the solution occurs (this effect is accompanied by a cor- 

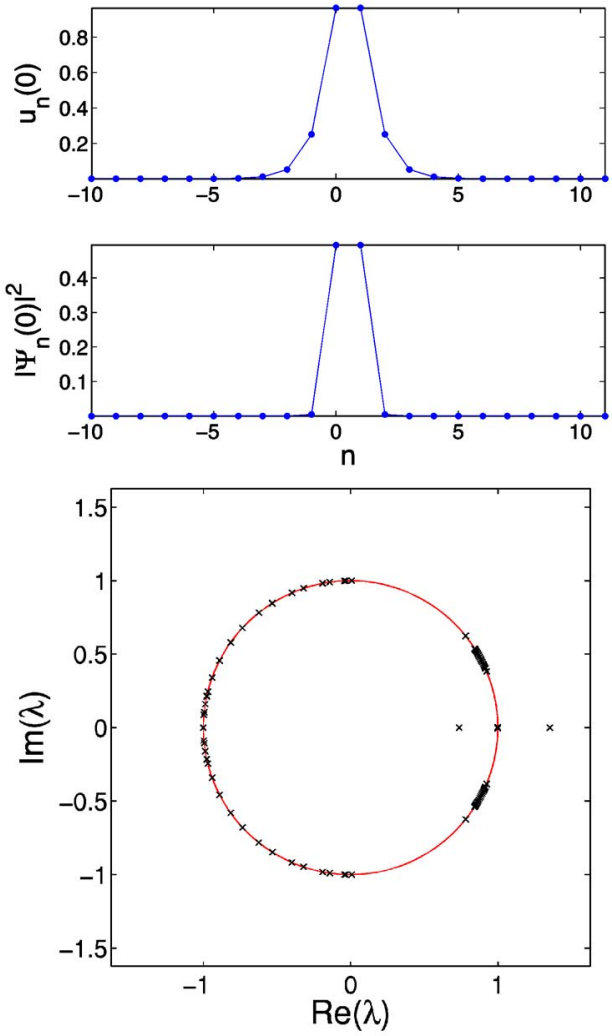

FIG. 11. (Color online) Top and middle panels: Profiles of the lattice ( $u_{n}$, top) and electronic component $\left(\left|\Psi_{n}\right|^{2}\right.$, middle) of a twosite polarobreather with $\omega_{\mathrm{b}}=0.8$ and $\chi=0.15$. Bottom panel: Floquet spectrum of this solution.

responding decrease of the energy of the immediate neighboring sites). Then, the system "relaxes" so that a quasibreathing evolution of the energy density is established; however, its strong localization at the central site of the lattice is essentially preserved for long times.

It is also possible to show the existence of a new branch of static polarons beyond $\chi_{c}$, as depicted in Fig. 20. This branch exists for $\chi \in(0.7,0.748)$ and the solutions are characterized by a large maximum amplitude and a linear spatial decay for $n<n_{c}$. For $n>n_{c}$ the lattice displacement decays exponentially. The quasibreathing behavior observed in Fig. 19 is due to the excitation of a solution belonging to this branch.

Beyond $\chi=0.748$, we have observed that seeding the lattice with a single-site localized initial condition leads to a continuous growth of the site amplitude. The energy density becomes more negative in the central site, whereas this is balanced by positive values of the energy density spreading along the lattice. These two effects balance each other in preserving the value of the total energy.

\section{CONCLUSIONS}

In this work, we have discussed the existence and stability of localized solutions in a generalized Holstein model with a soft (Morse-type) substrate potential. We have shown that, within this model, and in addition to the standard stationary
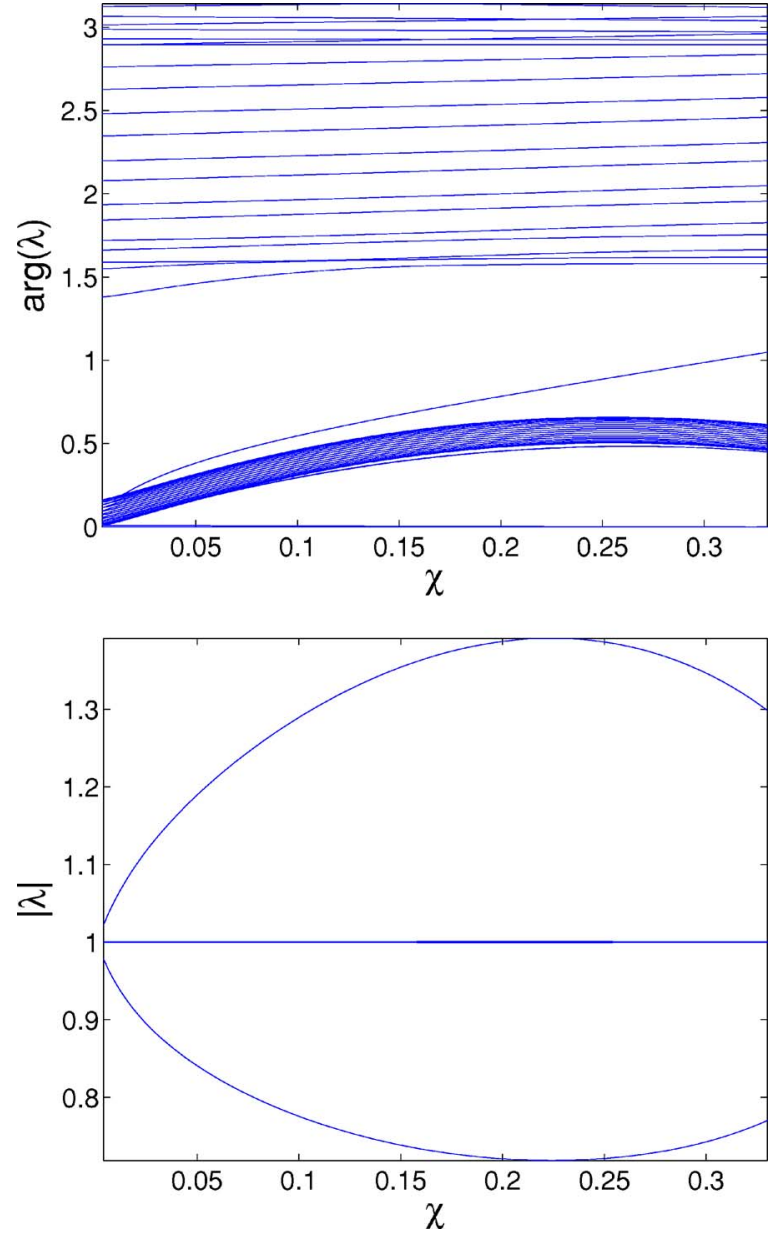

FIG. 12. (Color online) The argument (top) and modulus (bottom) of the Floquet eigenvalues as a function of the coupling constant $\chi$ for two-site polarobreathers.

polaronic solutions discussed in Ref. 12, there exists also a time-periodic type of state exhibiting a very robust breathing behavior, namely the polarobreather. Using Floquet analysis, we have elucidated the stability of these polarobreather solutions and we have also presented their domain of existence based on resonance conditions. In this respect, we have shown that the polarobreathers cease to exist as a result of their "collision" with the standard polaronic branch, occurring at frequency-dependent values of the relevant coupling parameter. We have also found that "phantom" versions of such polarobreathers exist in finite lattices but are usually unstable. Physically, we can view these dressed polaron solutions as "hot" polarons (or excitons in related excitonvibron systems). However, the polarobreather is dressed with a coherent wave packet (a breather), which binds with the polaron as a composite local excitation. We have also obtained multisite polarons (such as the two-site polarons examined herein) and multisite polarobreathers (such as the two-site ones, also studied herein), and numerically demonstrated their instability. Finally, we have shown that the unstable polaronic dynamics (for parameter values beyond the range of existence of their corresponding stationary branch) may lead to energy redistribution and eventual oscillatory dynamics on longer time scales. 

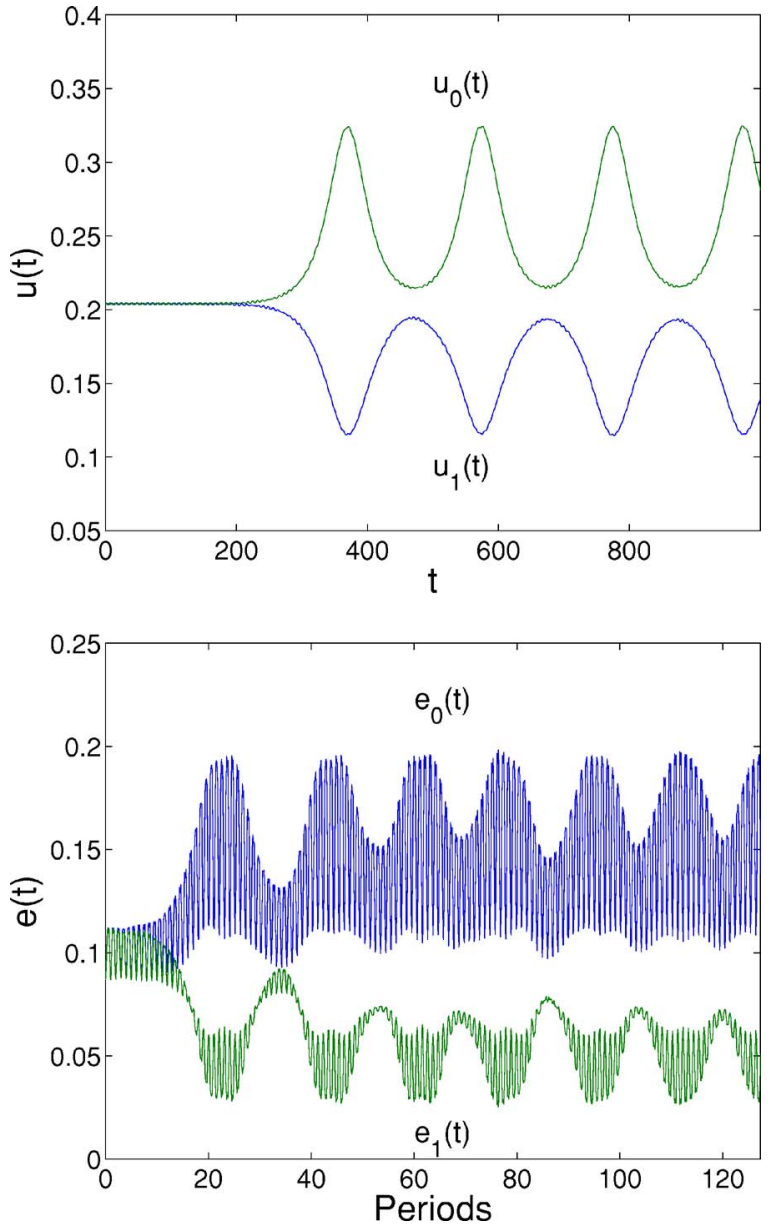

FIG. 13. (Color online) Evolution of the central particles' displacements and energy densities, respectively, for a perturbed intersite polaron with $\chi=0.35$ (top panel) and an intersite polarobreather with $\chi=0.2$ (bottom panel).

It will be interesting to examine higher-dimensional generalizations of the model and the possibility for obtaining localized solitary wave or vortex-like solutions in such higher-dimensional discrete contexts. Such studies are cur-
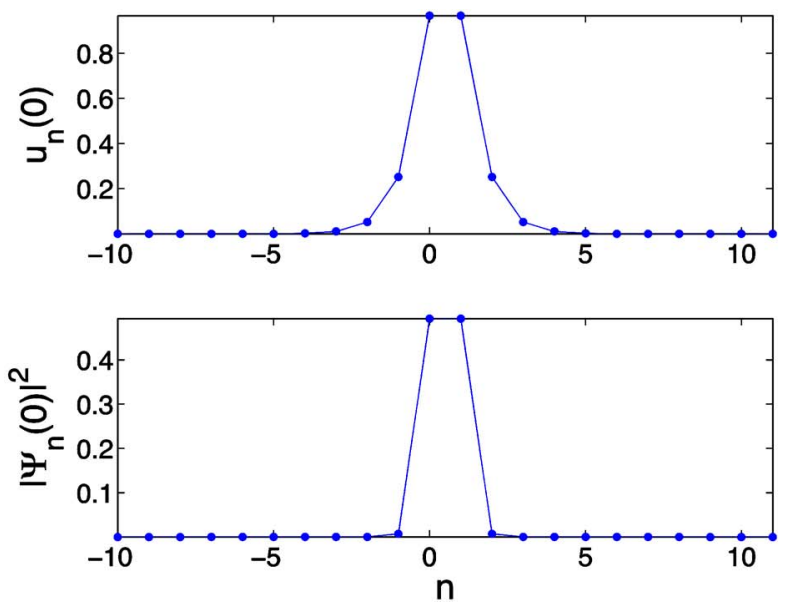

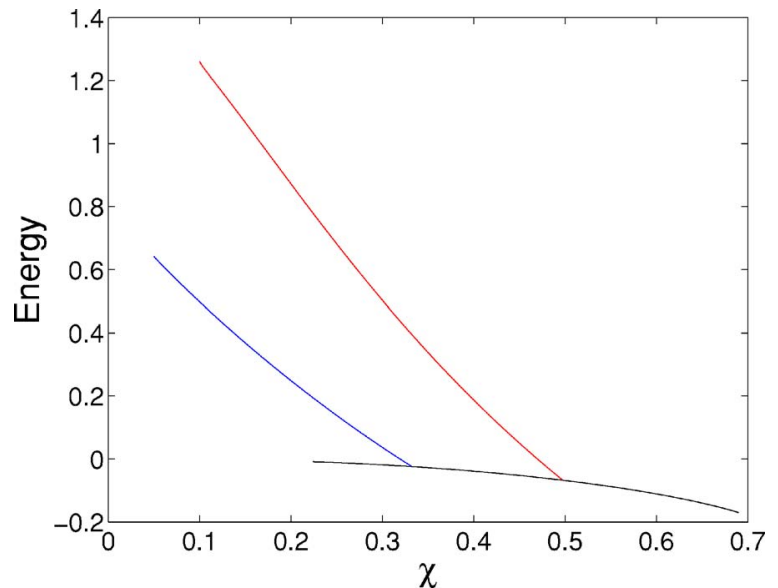

FIG. 15. (Color online) The energy of the twisted polarobreathers as a function of $\chi$ with $\omega_{\mathrm{b}}=0.8$. The longer (shorter) vertical line corresponds to type II (type I) solutions, and the bottom horizontal line to the static polaron.

rently in progress and will be reported elsewhere. It would also be important to assess the significance of quantum effects in both the electronic and lattice degrees of freedom. Initial results ${ }^{25}$ suggest that bound states of breathers and polarons (or excitons) may indeed persist in appropriate regimes.

Applications of the excitations we have identified include all polaron and exciton contexts where the induced charge or energy localization is strong enough, i.e., the small polaron/ exciton regime. This regime is the result of sufficiently strong coupling of the charge to the lattice degrees of freedom. Then, the lattice deformation induced around the selftrapped charge is also strongly localized (so that discreteness effects are dominant) and of large amplitude locally (so that anharmonic lattice effects can create an additional selftrapping of breather wave packets). Examples should include colossal magnetoresistant oxides, ${ }^{26}$ arrays of Bose-Einstein condensate droplets where the $s$-wave scattering length (i.e., the nonlinearity strength) is tuned to strong values, e.g., by Feshbach resonance, ${ }^{27}$ and so on. Similarly, situations in
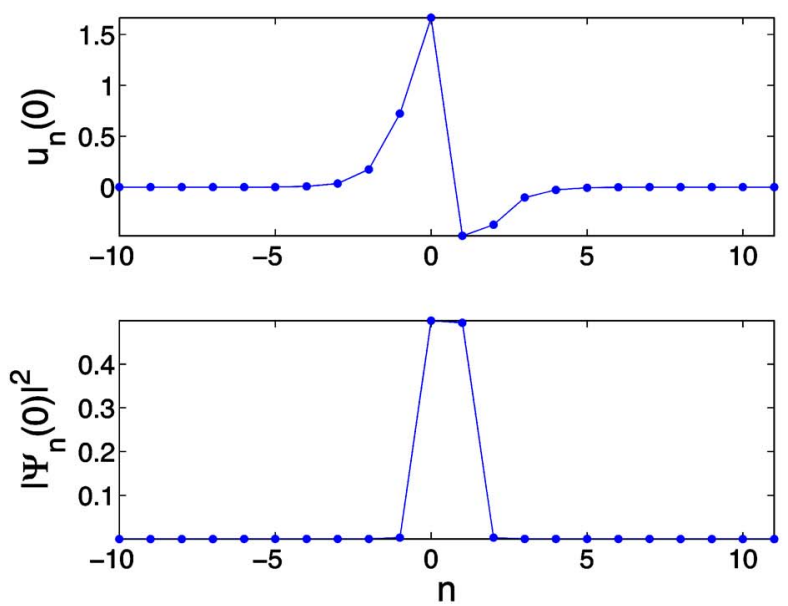

FIG. 14. (Color online) The profiles of the lattice (top) and the electronic (bottom) components of a type I (left panel) and a type II (right panel) twisted polarobreather for $\omega_{\mathrm{b}}=0.8$ and $\chi=0.15$. 

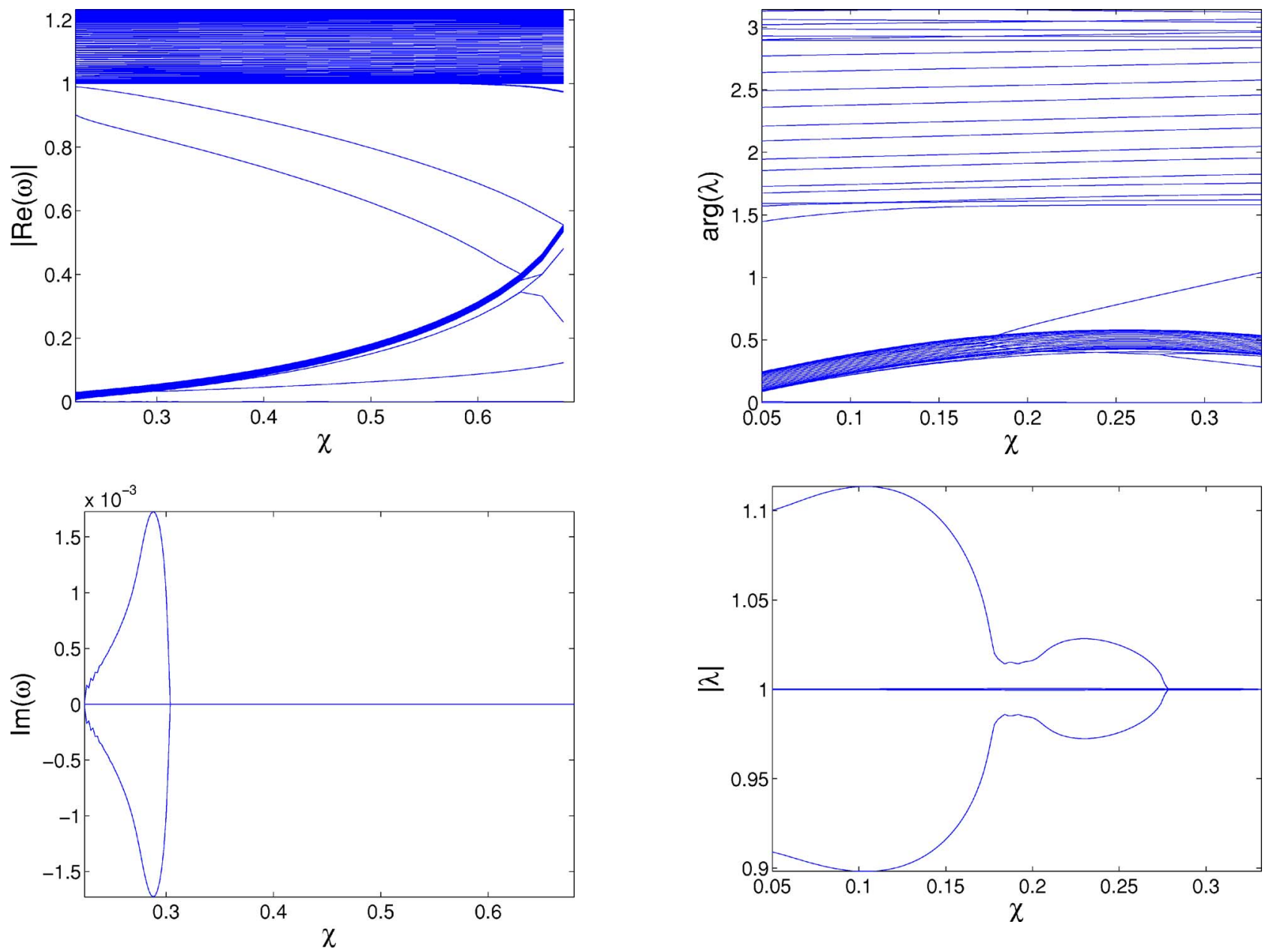

FIG. 16. (Color online) The real (top) and imaginary (bottom) parts of the linear mode spectrum as a function of the coupling constant $\chi$ for twisted polarons.

which charge injection takes place at high energy will lead to the same nonlinear lattice regime. This occurs, for example, with photoexcitation across large semiconducting or chargetransfer electronic gaps in materials such as conjugated polymers,${ }^{28}$ charge-transfer oxides,${ }^{29}$ and probably biological macromolecules.

In all such strong coupling situations, understanding and controlling the polarobreathers is of paramount importance, as they determine energy or charge localization and transport mechanisms. These mechanisms are fundamental to many device applications based on deformable electronic matter, as e.g., polymer LEDs.

\section{ACKNOWLEDGMENTS}

One of us (J.C.) acknowledges financial support from the MECD/FEDER project FIS2004-01183. P.G.K. acknowledges support from NSF-DMS-0204585, NSF-DMS0505663, and NSF-CAREER. Work at Los Alamos is performed under the auspices of the U.S. DOE.

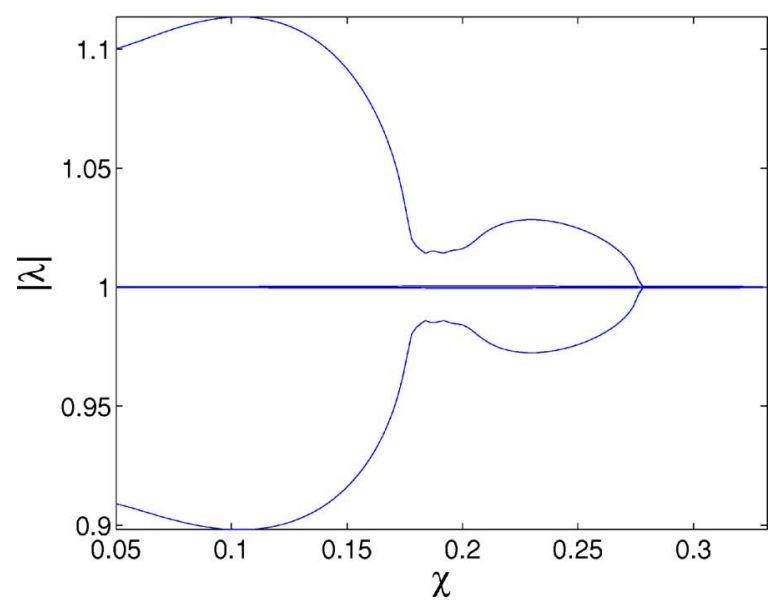

FIG. 17. (Color online) The argument (top) and modulus (bottom) of the Floquet eigenvalues as a function of the coupling constant $\chi$ for type I twisted polarobreathers.

\section{APPENDIX: DETAILS OF NUMERICAL METHODS}

\section{Real space methods}

In order to analyze Eqs. (6) and (7) using the real space method, it is convenient to separate the electronic wave function into its real and imaginary parts, $\Xi_{n}(t)=\phi_{n}(t)+i \varphi_{n}(t)$, and also define the oscillation velocity $v_{n}(t)=\dot{u}_{n}(t)$. In this way, we produce a system of $4 N$ equations ( $N$ is the number of particles of the system):

$$
\begin{gathered}
\dot{\phi}_{n}=-\left(-\omega_{e}+\chi u_{n}\right) \varphi_{n}-J\left(\varphi_{n+1}+\varphi_{n-1}\right), \\
\dot{\varphi}_{n}=\left(-\omega_{e}+\chi u_{n}\right) \phi_{n}+J\left(\phi_{n+1}+\phi_{n-1}\right), \\
\dot{u}_{n}=v_{n}, \\
\dot{v}_{n}=-V^{\prime}\left(u_{n}\right)+\chi\left(\phi_{n}^{2}+\varphi_{n}^{2}\right)+k\left(u_{n+1}-2 u_{n}+u_{n-1}\right) .
\end{gathered}
$$

Then, if we define $X(t) \equiv\left\{u_{n}(t), v_{n}(t), \phi_{n}(t), \varphi_{n}(t)\right\}$, there exists a map $\mathcal{T}$ which relates $X(0)$ and $X\left(T_{\mathrm{b}}\right)$ as $X\left(T_{\mathrm{b}}\right)=\mathcal{T} X(0)$. The shooting (real space) method consists in finding zeros of the map $F \equiv \mathcal{T X}(0)-X\left(T_{\mathrm{b}}\right)$ (Ref. 14) (for a detailed explana- 

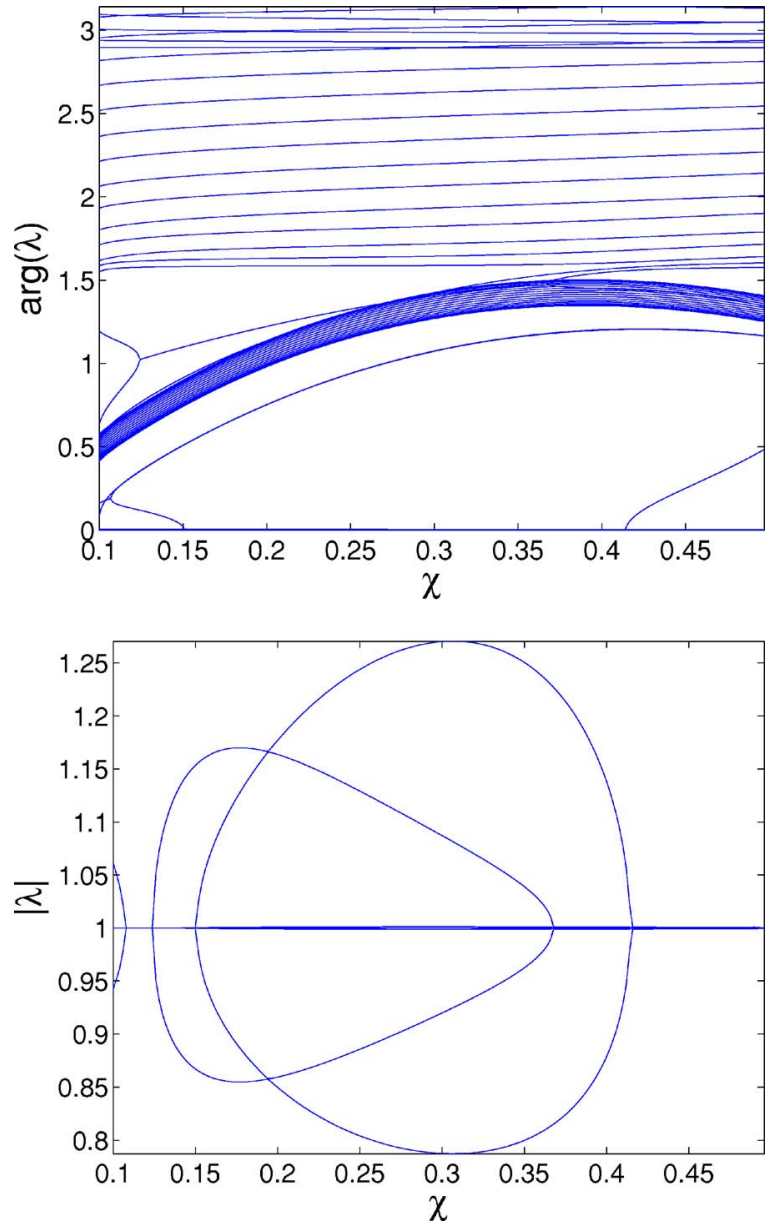

FIG. 18. (Color online) The argument (top) and modulus (bottom) of the Floquet eigenvalues as a function of the coupling constant $\chi$ for type II twisted polarobreathers.

tion of the shooting method, see Ref. 30). The tangent map needed for the application of the Newton method, $J \equiv \partial \mathcal{T}$, must be calculated numerically.

However, in this scheme the electronic norm is not conserved and the electronic frequency $\omega_{e}$ must be known. For this reason, an equation is added to Eq. (A1),

$$
\sum_{n}\left(\phi_{n}^{2}+\varphi_{n}^{2}\right)-1=0
$$

so that the norm is conserved, and a new variable $\omega_{e}$ is added to the vector $X(t)$.

\section{Fourier space methods}

The Fourier space methods (see details in Refs. 18 and 19) are based on the fact that both the electronic wave function and the lattice displacements are periodic with period $T_{\mathrm{b}}$. Thus they can be expressed in terms of a truncated Fourier series expansion:

$$
\Xi_{n}(t)=\sum_{k=-k_{m}}^{k_{m}} a_{n}^{k} \exp \left(i k \omega_{\mathrm{b}} t\right),
$$
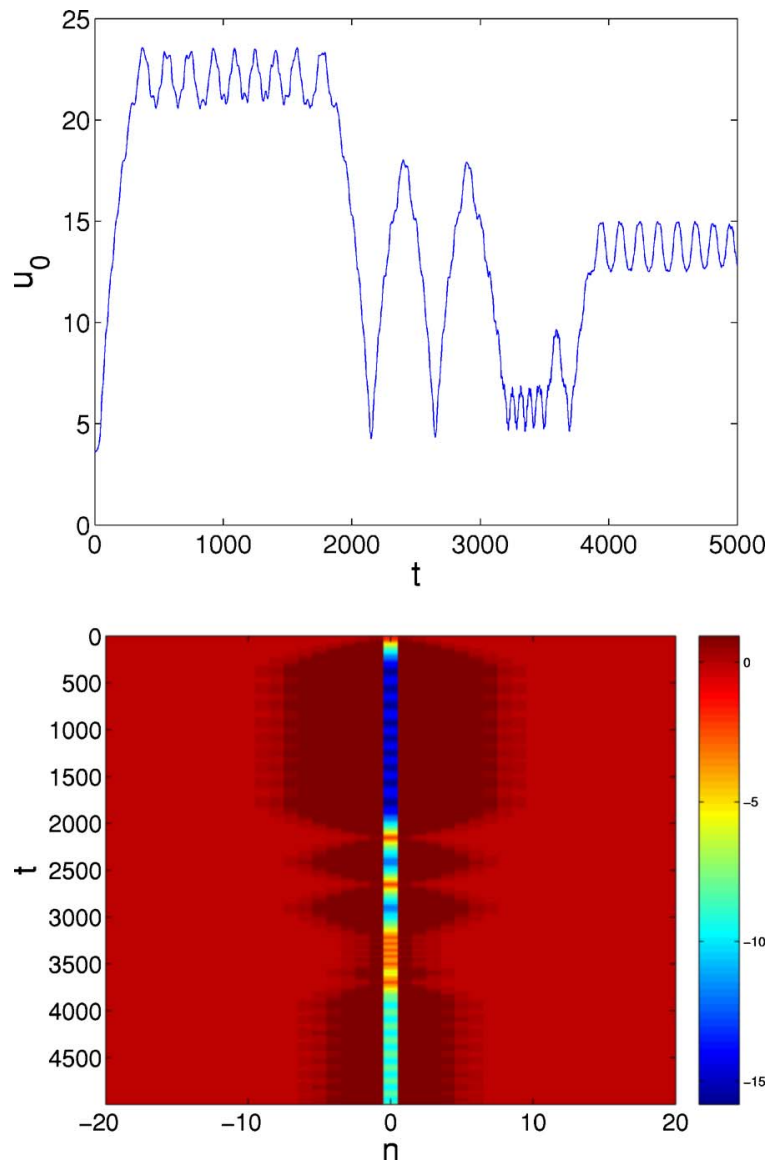

FIG. 19. (Color online) Top panel: Time evolution of the displacement of the central particle at $\chi=0.72$ using as the initial condition a static polaron at $\chi=0.718$. Bottom panel: Contour plot of the energy density.

$$
u_{n}(t)=\sum_{k=-k_{m}}^{k_{m}} b_{n}^{k} \exp \left(i k \omega_{\mathrm{b}} t\right) .
$$

Then, the dynamical equations (6) and (7) are reduced to a set of $(2 N+1) \times\left(2 k_{m}+1\right)$ algebraic equations (we have also included the norm conservation), where the variables are $Z$ $\equiv\left\{a_{n}^{k}, b_{n}^{k}, \omega_{e}\right\}$ :

$$
\begin{gathered}
F_{E, n}^{k} \equiv-\omega_{\mathrm{b}} k a_{n}^{k}-\omega_{e} a_{n}^{k}+\mathcal{F}^{k}\left[V^{\prime}\left(u_{n}\right)\right]+\chi \mathcal{F}^{k}\left(u_{n} \Xi_{n}\right) \\
+J\left(a_{n-1}^{k}+b_{n+1}^{k}\right)=0, \\
F_{B, n}^{k} \equiv-\omega_{\mathrm{b}}^{2} k^{2} b_{n}^{k}+\mathcal{F}^{k}\left[V^{\prime}\left(u_{n}\right)\right]-\chi \mathcal{F}^{k}\left(\left|\Xi_{n}\right|^{2}\right) \\
-k\left(b_{n-1}^{k}-2 b_{n}^{k}+b_{n+1}^{k}\right)=0, \\
F_{N, n} \equiv\left(\sum_{n} \sum_{k} \sum_{k^{\prime}} a_{n}^{k} a_{n}^{k^{\prime}}\right)-1=0 .
\end{gathered}
$$

In the above equations, $\mathcal{F}^{k}$ denotes the discrete Fourier transform: 

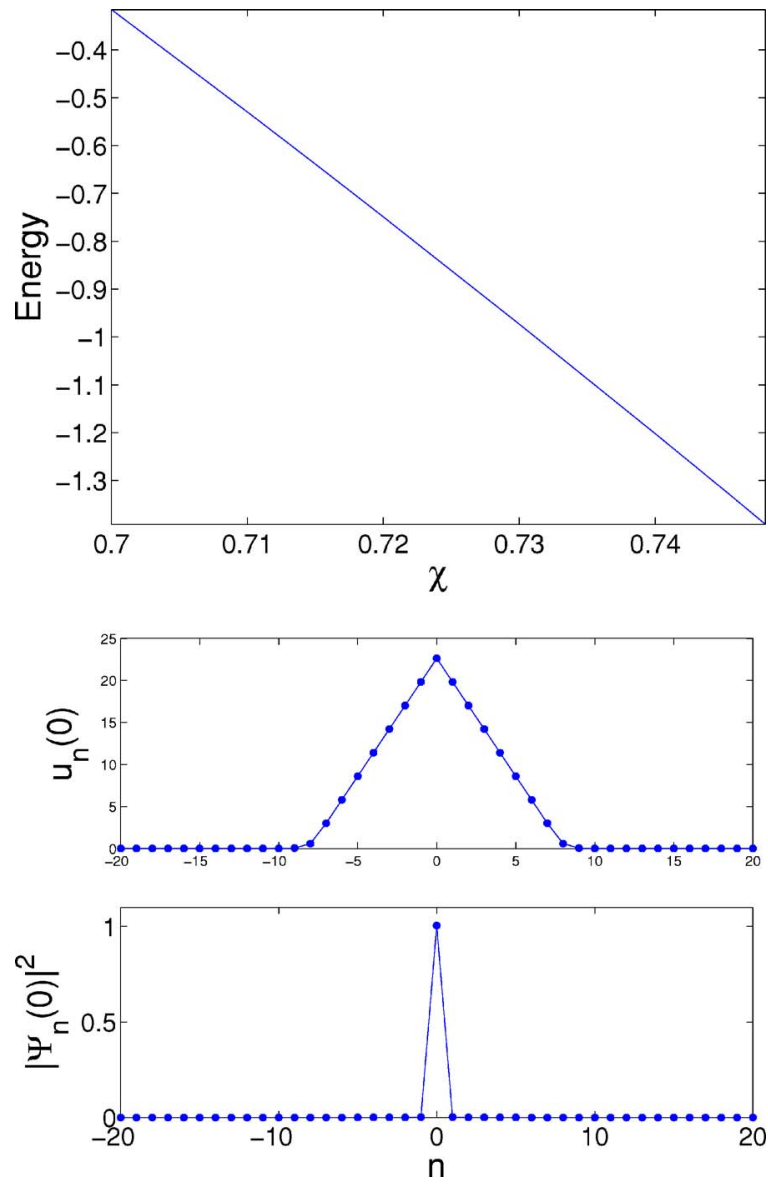

FIG. 20. (Color online) Top panel: Energy as a function of the coupling constant $\chi$ for the new branch of polarons. Middle and bottom panels: Lattice (middle) and electronic (bottom) coordinates of a polaron of this branch.

$$
\mathcal{F}^{k}(u)=\sum_{j=-k_{m}}^{k_{m}} u\left(t_{j}\right) \exp \left(i k \omega_{\mathrm{b}} t_{j}\right),
$$

where $t_{j}$ is a sample of times that must be equally spaced according to

$$
t_{j}=\frac{2 \pi j}{\omega_{\mathrm{b}}\left(2 k_{m}+1\right)}, \quad j=-k_{m}, \ldots,+k_{m},
$$

and $u\left(t_{j}\right)$ is calculated from the Fourier coefficients $b^{k}$ by means of the inverse discrete Fourier transform:

$$
u\left(t_{j}\right)=\sum_{k=-k_{m}}^{k_{m}} b^{k} \exp \left(i k \omega_{\mathrm{b}} t_{j}\right) .
$$

The Newton operator or Jacobian $J \equiv \partial F$ (with $F$ $\left.\equiv\left\{F_{P, n}^{k} ; F_{B, n}^{k} ; F_{N}\right\}\right)$ can be calculated analytically. One of the main disadvantages of this method is that the Jacobian is singular. Polarobreathers must be calculated using singular value decomposition. ${ }^{31}$ However, this method allows the calculation of non-time-reversible solutions.

\section{Normal modes and linear stability analysis}

Here, we present the normal modes and stability equations, which were used in the results presented in the main text.

\section{a. Stationary solution stability: Normal modes}

In order to calculate the normal modes we first need to calculate a stationary polaron from Eqs. (1) and (2) from the following conditions: ${ }^{12}$

$$
\begin{gathered}
\Psi_{n}(t)=\psi_{n} \exp \left(-i \omega_{e} t\right), \\
\ddot{u}_{n}(t)=\dot{u}_{n}(t)=0 .
\end{gathered}
$$

Then, the polaron is determined by the electronic wave function $\psi_{n}$ and the lattice displacements $y_{n}$.

The normal modes are introduced as perturbations to a stationary polaron: ${ }^{8,13,17}$

$$
\begin{gathered}
\Psi_{n}(t)=\left[\psi_{n}+\epsilon_{n}(t)\right] \exp \left(-i \omega_{e} t\right), \\
u_{n}(t)=y_{n}+\xi_{n}(t), \\
\dot{u}_{n}(t)=\dot{y}_{n}+\pi_{n}(t)=\pi_{n}(t),
\end{gathered}
$$

and the dynamical equations for the normal modes are

$$
\begin{gathered}
i \dot{\epsilon}_{n}=-\omega_{e} \epsilon_{n}-\chi\left(y_{n} \epsilon_{n}+\psi_{n} \xi_{n}\right)-J\left(\epsilon_{n+1}+\epsilon_{n-1}\right), \\
\dot{\xi}_{n}=\pi_{n} \\
\dot{\pi}_{n}=-V^{\prime \prime}\left(y_{n}\right) \xi_{n}+\chi \psi_{n}\left(\epsilon_{n}+\epsilon_{n}^{*}\right) \\
+k\left(\xi_{n+1}-2 \xi_{n}+\xi_{n-1}\right) .
\end{gathered}
$$

Normal mode frequencies can be calculated using the following relations:

$$
\begin{aligned}
& \epsilon_{n}(t)=a_{n} \exp (i \omega t)+b_{n} \exp \left(-i \omega^{*} t\right) \\
& \xi_{n}(t)=c_{n} \exp (i \omega t)+c_{n}^{*} \exp \left(-i \omega^{*} t\right) \\
& \pi_{n}(t)=d_{n} \exp (i \omega t)+d_{n}^{*} \exp \left(-i \omega^{*} t\right)
\end{aligned}
$$

Thus the normal modes are determined by the following linear system:

$$
\begin{gathered}
\omega a_{n}=\omega_{e} a_{n}+\chi\left(y_{n} a_{n}+\psi_{n} c_{n}\right)+J\left(a_{n+1}+a_{n-1}\right), \\
\omega b_{n}^{*}=-\omega_{e} b_{n}^{*}-\chi\left(y_{n} b_{n}^{*}+\psi_{n} c_{n}\right)-J\left(b_{n+1}^{*}+b_{n-1}^{*}\right), \\
\omega c_{n}=-i d_{n}, \\
\omega d_{n}=i\left[V^{\prime \prime}\left(y_{n}\right) c_{n}-\chi \psi_{n}\left(a_{n}+b_{n}^{*}\right)+k\left(c_{n+1}-2 c_{n}+c_{n-1}\right)\right] .
\end{gathered}
$$

As the normal mode operator is non-Hermitian, the frequencies are in general complex numbers. If nonreal frequencies exist in the spectrum, then the stationary polaron is unstable. 


\section{b. Polarobreather stability: Floquet spectrum}

Linear stability can be studied via a Floquet analysis. This analysis is performed by linearizing the dynamical equations around a polarobreather. Then, the analysis performed for the normal modes cannot be extended to the stability, as the electronic wave function does not possess gauge invariance. Thus we introduce a perturbation $\Omega \equiv\left\{\alpha_{n}, \beta_{n}, \xi_{n}, \pi_{n}\right\}$ to Eqs. (A1), which has the form

$$
\phi_{n}=\phi_{n}^{0}+\alpha_{n}, \quad \varphi_{n}=\varphi_{n}^{0}+\beta_{n}, \quad u_{n}=u_{n}^{0}+\xi_{n}, \quad \pi_{n}=\dot{\xi}_{n},
$$

where the superscript zero denotes the polarobreather solution. Then, to leading-order approximation (i.e., keeping only the linear terms), the stability equations are

$$
\begin{gathered}
\dot{\alpha}_{n}=-\left(-\omega_{e}+\chi u_{n}^{0}\right) \beta_{n}-\chi \varphi_{n}^{0} \xi_{n}-J\left(\alpha_{n+1}+\alpha_{n-1}\right), \\
\dot{\beta}_{n}=\left(-\omega_{e}+\chi u_{n}^{0}\right) \alpha_{n}+\chi \phi_{n}^{0} \xi_{n}+J\left(\beta_{n+1}+\beta_{n-1}\right), \\
\dot{\xi}_{n}=\pi_{n}, \\
\dot{\pi}_{n}=2 \chi\left(\phi_{n}^{0} \alpha_{n}+\varphi_{n}^{0} \beta_{n}\right)-V^{\prime \prime}\left(u_{n}^{0}\right)+k\left(\xi_{n+1}-2 \xi_{n}+\xi_{n-1}\right) .
\end{gathered}
$$

The Floquet operator (or monodromy matrix) $\mathcal{M}$ relates the vector $\Omega$ at $t=0$ and $t=T_{\mathrm{b}}$ as follows:

$$
\Omega\left(T_{\mathrm{b}}\right)=\mathcal{M} \Omega(0) .
$$

A polarobreather is stable if all the eigenvalues of the monodromy matrix lie on the unit circle.
${ }^{1}$ S. Aubry, Physica D 103, 201 (1997); S. Flach and C. R. Willis, Phys. Rep. 295, 181 (1998); D. Hennig and G. Tsironis, ibid. 307, 333 (1999); P. G. Kevrekidis, K. Ø. Rasmussen, and A. R. Bishop, Int. J. Mod. Phys. B 15, 2833 (2001).

${ }^{2}$ D. N. Christodoulides, F. Lederer, and Y. Silberberg, Nature (London) 424, 817 (2003); Yu. S. Kivshar and G. P. Agrawal, Optical Solitons: From Fibers to Photonic Crystals (Academic Press, San Diego, CA, 2003).

${ }^{3}$ P. G. Kevrekidis and D. J. Frantzeskakis, Mod. Phys. Lett. B 18, 173 (2004); V. V. Konotop and V. A. Brazhnyi, ibid. 18, 627 (2004); P. G. Kevrekidis, R. Carretero-González, D. J. Frantzeskakis, and I. G. Kevrekidis, ibid. 18, 1481 (2004).

${ }^{4}$ M. Sato and A. J. Sievers, Nature (London) 432, 486 (2004); M. Sato, B. E. Hubbard, A. J. Sievers, B. Ilic, and H. G. Craighead, Europhys. Lett. 66, 318 (2004); M. Sato, B. E. Hubbard, A. J. Sievers, B. Ilic, D. A. Czaplewski, and H. G. Craighead, Phys. Rev. Lett. 90, 044102 (2003).

${ }^{5}$ M. Peyrard, Nonlinearity 17, R1 (2004).

${ }^{6}$ N. Voglis, Mon. Not. R. Astron. Soc. 344, 575 (2003).

${ }^{7}$ T. Holstein, Ann. Phys. (N.Y.) 8, 325 (1959).

${ }^{8}$ G. Kalosakas, S. Aubry, and G. P. Tsironis, Phys. Rev. B 58, 3094 (1998).

${ }^{9}$ S. Aubry, G. Abramovici, and J.-L. Raimbault, J. Stat. Phys. 67, 675 (1992).

${ }^{10}$ A. S. Davydov, J. Theor. Biol. 38, 559 (1973); 66, 379 (1977).

${ }^{11}$ A. C. Scott, Phys. Rep. 217, 1 (1992).

${ }^{12}$ M. A. Fuentes, P. Maniadis, G. Kalosakas, K. Ø. Rasmussen, A. R. Bishop, V. M. Kenkre, and Yu. B. Gaididei, Phys. Rev. E 70, 025601(R) (2004).

${ }^{13}$ P. Maniadis, G. Kalosakas, K. Ø. Rasmussen, and A. R. Bishop, Phys. Rev. B 68, 174304 (2003).

${ }^{14}$ G. Kalosakas and S. Aubry, Physica D 113, 228 (1998).
${ }^{15}$ R. S. MacKay and S. Aubry, Nonlinearity 7, 1623 (1994).

${ }^{16}$ J. L. Marín and S. Aubry, Nonlinearity 9, 1501 (1996).

${ }^{17}$ N. K. Voulgarakis and G. P. Tsironis, Phys. Rev. B 63, 014302 (2000).

${ }^{18}$ J. F. R. Archilla, R. S. MacKay, and J. L. Marín, Physica D 136, 406 (1999).

${ }^{19}$ J. Cuevas, J. F. R. Archilla, F. Palmero, and F. Romero, J. Phys. A 34, L221 (2001).

${ }^{20}$ J. Cuevas, C. Katerji, J. F. R. Archilla, J. C. Eilbeck, and F. M. Russel, Phys. Lett. A 315, 364 (2003).

${ }^{21}$ J. Cuevas, J. F. R. Archilla, B. Sánchez-Rey, and F. R. Romero, Physica D 216, 115 (2006).

${ }^{22}$ A. M. Morgante, M. Johansson, S. Aubry, and G. Kopidakis, J. Phys. A 35, 4999 (2002).

${ }^{23}$ J. Cuevas, J. F. R. Archilla, Y. B. Gaididei, and F. R. Romero, Physica D 163, 106 (2002).

${ }^{24}$ D. E. Pelinovsky, P. G. Kevrekidis, and D. J. Frantzeskakis, Physica D 212, 1 (2005).

${ }^{25}$ W. Z. Wang, A. R. Bishop, J. T. Gammel, and R. N. Silver, Phys. Rev. Lett. 80, 3284 (1998).

${ }^{26}$ H. Röder, Jun Zang, and A. R. Bishop, Phys. Rev. Lett. 76, 1356 (1996).

${ }^{27}$ S. Inouye, M. R. Andrews, J. Stenger, H. J. Miesner, D. M. Stamper-Kurn, and W. Ketterle, Nature (London) 392, 151 (1998); J. L. Roberts, N. R. Claussen, J. P. Burke, Jr., C. H. Greene, E. A. Cornell, and C. E. Wieman, Phys. Rev. Lett. 81, 5109 (1998).

${ }^{28}$ S. Tretiak, A. Saxena, R. L. Martin, and A. R. Bishop, Proc. Natl. Acad. Sci. U.S.A. 100, 2185 (2003).

${ }^{29}$ A. Bussmann-Holder, A. R. Bishop, and T. Egami, Europhys. Lett. 71, 249 (2005).

${ }^{30}$ J. L. Marín, Ph.D. Thesis, University of Zaragoza, 1997.

${ }^{31}$ T. Cretegny and S. Aubry, Phys. Rev. B 55, R11929 (1997). 